Surface Science 601 (2007) 4603

\title{
Growth of thin alumina films on a vicinal $\mathrm{NiAl}$ surface
}

\author{
Stefan Ulrich, Niklas Nilius*, Hans-Joachim Freund \\ Fritz-Haber-Institut der MPG \\ Berlin, Germany
}

\begin{abstract}
Dramatic changes in the surface morphology have been observed during the oxidation of stepped $\operatorname{NiAl}(16,14,1)$ by LEED and STM. The initial sequence of identical $(110)$ terraces is lifted in favor of large, triangular planes, whose mean size is determined by the mismatch-induced stress that accumulates in the thin alumina film. The asymmetry of the original step direction on $\operatorname{NiAl}(16,14,1)$ with respect to the orientation of the two alumina reflection domains favors the formation of one domain type, for which the stress relief via NiAl step edges is particularly efficient.

* Corresponding author: nilius@fhi-berlin.mpg.de
\end{abstract}

Self-organization on the nanometer scale provides a powerful means to produce assemblies of identical entities with well-defined properties, thus opening an efficient way to design new, functionalized materials. The self-organization can be initiated by material deposition onto template surfaces offering an ordered array of binding sites [1,2,3,4], or by exploiting adsorbate-adsorbate interactions that lead to the development of super-structures $[5,6]$. Template surfaces are fabricated in various ways, for instance (i) by cutting a crystal along a high index plane to form a regular step pattern [7,8], (ii) by growing over-layers with small lattice mismatch with the support to induce stress-related relaxation networks $[1,4]$ or (iii) by utilizing long-ranged electronic modulations originating from electronic superstructures or charge-density waves $[9,10]$. Template surface that are chemically inert are of special interest, as they only weakly alter the intrinsic properties of the ad-material. Considerable efforts have therefore been made to fabricate nano-patterned oxide surfaces that minimize the adsorbate-support interactions due to their band gap [11,12].

This work reports on the growth of ultra-thin alumina films on a stepped NiAl surface. The alumina film on flat $\mathrm{NiAl}(110)$ belongs to the best-explored metal-oxide systems, and a detailed picture of its stoichiometry, atomic structure and electronic properties has evolved from extended experimental and theoretical investigations $[13,14,15]$. The film exhibits a well-defined defect structure, consisting of a network of dislocation lines. These surface defects are responsible for various chemical and physical properties of the oxide, such as the nucleation of metal adatoms or the dissociation of molecules $[14,16]$. The experiments described in this work aim for a controlled modification of the defect structure by growing the alumina film on a vicinal NiAl surface, where intrinsic dislocation lines might be replaced by the regular step pattern. Manipulating the defect structure of an oxide surface is 
of scientific and technological relevance, because it opens a way to fabricate arrays of suited nucleation sites for the growth of metal particle or nano-wires, which can be used as model systems for catalytic and magnetic investigations. Also intrinsic oxide properties might be controlled by changing the abundance of one specific defect type, e.g. of opticallyactive color or paramagnetic spin centers $[11,17]$.

Preparation of the alumina film on flat and vicinal NiAl surfaces involves exposing the surface to $1200 \mathrm{~L} \mathrm{O}_{2}$ at $550 \mathrm{~K}$ and annealing to $1000 \mathrm{~K}$, as described in the literature [13]. Partially oxidized surfaces are produced by reducing the $\mathrm{O}_{2}$ dose to 60-90 L. The structural properties of the sample before and after film formation are determined by STM and LEED, whereby a liquid-nitrogen cooled, beetle-type microscope is used for the STM experiments. On flat $\mathrm{NiAl}(110)$, the $0.5 \mathrm{~nm}$ thin alumina film grows in two reflection domains $(\mathrm{A}, \mathrm{B})$ that are tilted by $\pm 24^{\circ}$ with respect to the [110] direction of the support (Fig.1A) [14,18]. Reflection (between domain A and B) and antiphase domains boundaries (APDB) (between $\mathrm{A}-\mathrm{A}$ and B-B) are the main structural defect of the film. Due to their oxygen-deficient nature, the dislocation lines induce a set of unoccupied states in the alumina band gap, which leads to their bright appearance in STM images taken at positive sample bias [19,20]. Whereas the reflection domain boundaries occur randomly on the surface, the APDB form a relatively regular pattern of dislocation lines that mainly run along the short axis of the two oxide unit cells [21]. APDB are introduced every 8-10 $\mathrm{nm}$ into the perfect oxide lattice to release stress and strain in the film that accumulates due to the lattice mismatch with the $\mathrm{NiAl}$ along the [110] direction. Along [001], metal and oxide overlayer grow incommensurate, which inhibits the development of lattice strain.

For the preparation of patterned alumina films, a $\mathrm{NiAl}(16,14,1)$ surface is used, which is a vicinal to the (110) plane tilted by $4.7^{\circ}$ along the [111] direction. The miscut angle induces a periodic succession of monatomic steps running at an angle of $34^{\circ}$ with respect to the [001] direction (Fig.1A,B). The $(16,14,1)$ surface plane is chosen to favor the development of one of the two alumina domains, as demonstrated below. An STM topographic image of vicinal $\operatorname{NiAl}(16,14,1)$ before oxidation is shown in Fig.2A. The surface exhibits the expected succession of (110) terraces of $(2.5 \pm 0.5) \mathrm{nm}$ width. Step edges appear with ragged shape, resulting from an alteration of short sections running along [001] and [111] direction (Fig.1B). Apparently, these directions mark the low-energy step orientations on the (110) surface and therefore develop at thermodynamic equilibrium conditions. Only the mean step orientation, tilted by $34^{\circ}$ with respect to $\mathrm{NiAl}$ [001], follows the value expected from the miscut angle. The morphology of the clean surface derived from STM is confirmed by LEED measurements, which exhibit the distinct splitting of fundamental spots compatible with the presence of a regular step pattern with $2.5 \mathrm{~nm}$ lattice constant (Fig. 1C). 


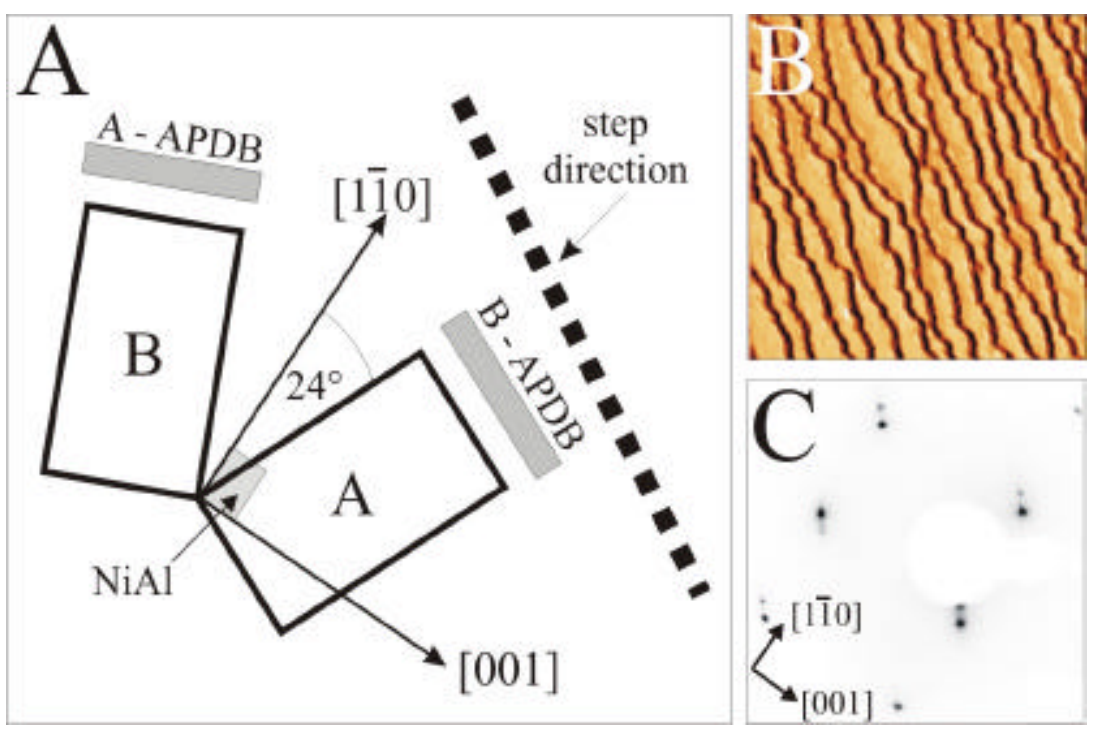

Fig.1

(A) Structural model of the alumina film on $\mathrm{NiAl}(110)$ showing the unit cells of the reflection domains, the major direction of antiphase domain boun-daries (APDB) and the step orientation on the vicinal $\operatorname{NiAl}(16,14,1)$.

(B) STM image $(40 \times 40$ $\mathrm{nm}^{2}, \mathrm{U}_{\text {Sample }}=0.85 \mathrm{~V}, \mathrm{I}=$ $0.6 \mathrm{nA}$ ) and (C) LEED of clean $\mathrm{NiAl} \quad(16,14,1)$ exhibiting the regular step pattern.

Already small amounts of $\mathrm{O}_{2}(60 \mathrm{~L})$ dosed onto $\mathrm{NiAl}(16,14,1)$ followed by annealing to $1000 \mathrm{~K}$ initiate dramatic changes in the surface morphology (Fig.2B). The succession of identical (110) terraces disappears on the expense of extended (110) planes, which emerge in alteration with small-sized terraces. The resulting terrace-width distribution exhibits a bimodal shape that can be fitted by two Gaussians peaking at $1.5 \mathrm{~nm}(\sigma=0.2 \mathrm{~nm})$ and $9.8 \mathrm{~nm}$ $(\sigma=8.1 \mathrm{~nm})$ for narrow and broad terraces, respectively (Fig.2E). Large (110) planes are often delimited by step bunches containing ten and more single steps, which are not observed on the clean NiAl surface. They also exhibit characteristic triangular shapes, originating from the interplay of straight [001] oriented steps and ragged boundaries with roughly [111] orientation. In well-resolved STM images, metallic surface regions are clearly distinguished from areas covered by alumina patches due to the characteristic line pattern of the alumina lattice (Fig.3A,C). At $60 \mathrm{~L} \mathrm{O}_{2}$ exposure, the oxidized fraction of the surface is approximated to $20 \%$. Despite their small size, the alumina patches show the well-known crystalline structure and no disordered oxide phase occurs on the surface. Oxide islands exclusively nucleate at the topside of step edges, and either cover small sections of large (110) planes or blanket small-size terraces (Fig.3B,C). Occasionally, extended stripes of $2 \mathrm{~nm}$ width and $0.2 \mathrm{~nm}$ height are found on the surface, that strictly follow the $\mathrm{NiAl}[001]$ direction and border the triangular (110) planes (see arrows in Fig.3A).

With increasing oxygen dose, further changes in the morphology are observed, until complete surface oxidation is achieved at around 500L O $\mathrm{O}_{2}$ exposure (Fig.2C). The dominant structures are now large (110) planes that are more uniform in size and shape than after incomplete oxidation. Correspondingly, terraces with less than $2 \mathrm{~nm}$ width cover a much smaller fraction of the surface. The terrace width-distribution retains therefore its bimodal character, with peak positions shifted to $1.1 \mathrm{~nm}(\sigma=0.3 \mathrm{~nm})$ and $7.9 \mathrm{~nm}(\sigma=5.0 \mathrm{~nm})$ for 
narrow and wide terraces, respectively (Fig.2F). Height profiles across a fully-oxidized $(16,14,1)$ surface indicate the presence of step bunches, containing approximately 10 single steps, while monatomic steps have more or less disappeared. Even after complete oxidation, dislocation lines between neighboring oxide domains are rarely observed on the vicinal surface, although they dominate STM images of the alumina film on flat $\mathrm{NiAl}(110)$ (Fig.4B).
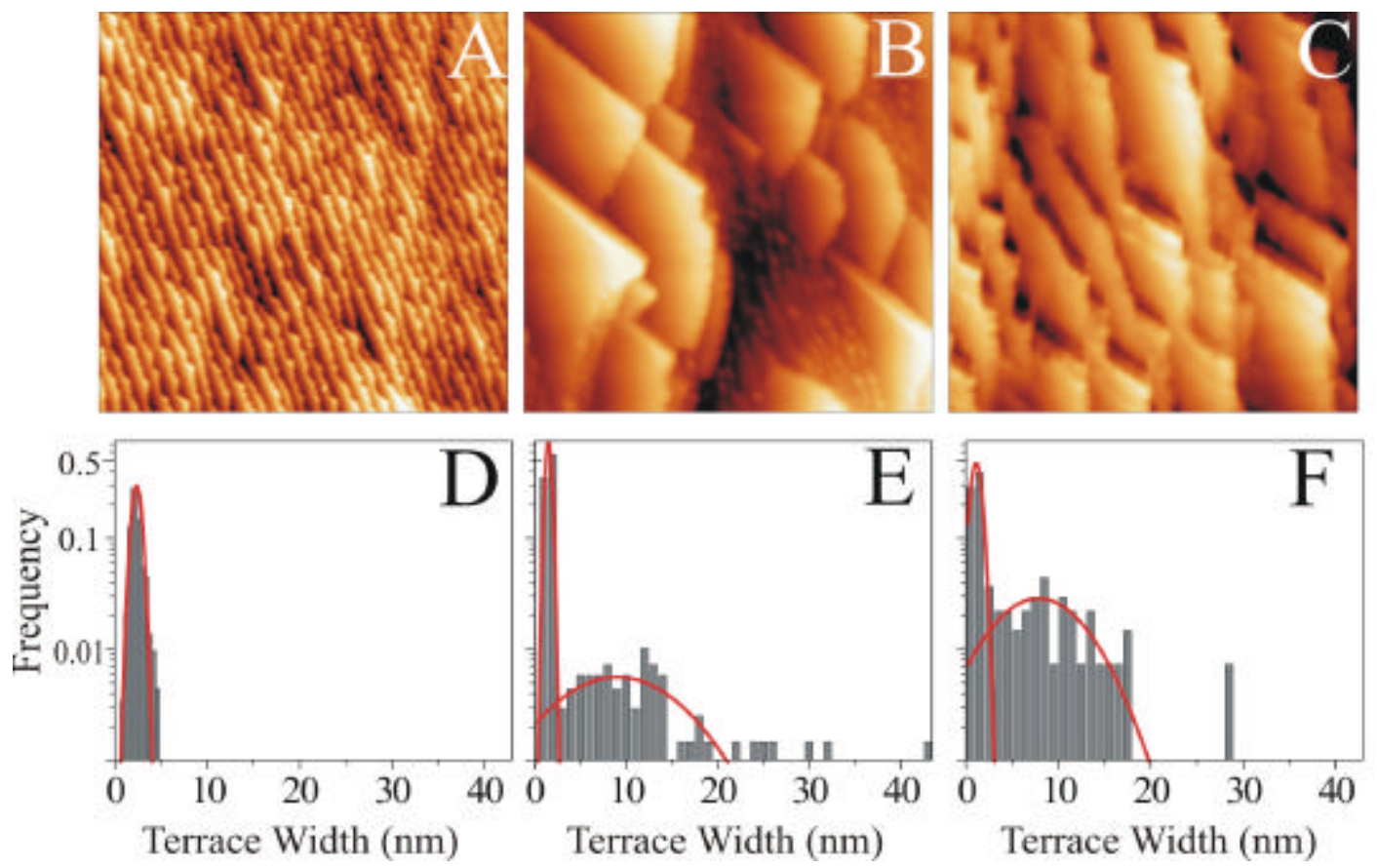

Fig.2

STM topographic images of $\mathrm{NiAl}(16,14,1)$ : (A) clean surface, $(\mathbf{B})$ after exposure to $60 \mathrm{~L} \mathrm{O}_{2}$ and (C) after $1200 \mathrm{~L} \mathrm{O}_{2}$ dosage $\left(200 \times 200 \mathrm{~nm}^{2}, \mathrm{U}_{\text {Sample }}=4.5 \mathrm{~V}, \mathrm{I}=0.1 \mathrm{nA}\right)$. While terraces on the clean metal are delimited by monatomic steps, step bunches of 2.0-2.5 nm height occur after oxygen treatment. (D-F) Terrace width distribution for the different oxidation stages as derived from STM images. The lines depict Gaussian fits to the data.

The drastic changes in surface morphology observed during oxidation of $\operatorname{NiAl}(16,14,1)$ demonstrate the strong metal-oxide interactions accompanying the growth of the alumina film. From STM images taken at small oxygen exposure, the topside of step edges is identified as the thermodynamically favored nucleation site for oxide islands (Fig.3). A similar nucleation behavior was observed for other oxide materials before [22,23]. Several reasons might be responsible for the initialization of the oxidation process on-top of a step edge: (i) Atoms at step positions have a lower local coordination than those incorporated into terraces and are usually more reactive. (ii) During oxidation of NiAl towards alumina, $\mathrm{Ni}$ atoms have to be removed from the reaction site, which normally occurs via diffusion into the bulk. On step edges, the energy-expensive sub-surface diffusion of Ni might be replaced by the less-activated surface diffusion, favoring the initial oxidation of steps. (iii) 
The electron-density at the topside of an edge is depleted and charge accumulates at its bottom, which leads to a flatter electron distribution across a surface step following the Smucholowski effect. This electron deficiency at the upper terrace facilitates the anchoring of the oxide layer, since this involves charge donation from the interfacial $\mathrm{Al}_{\text {oxide }}$ atoms towards the NiAl support [15]. The oxide layer therefore behaves like a nucleophilic molecule that also preferentially binds to the topside of a step edge [24].

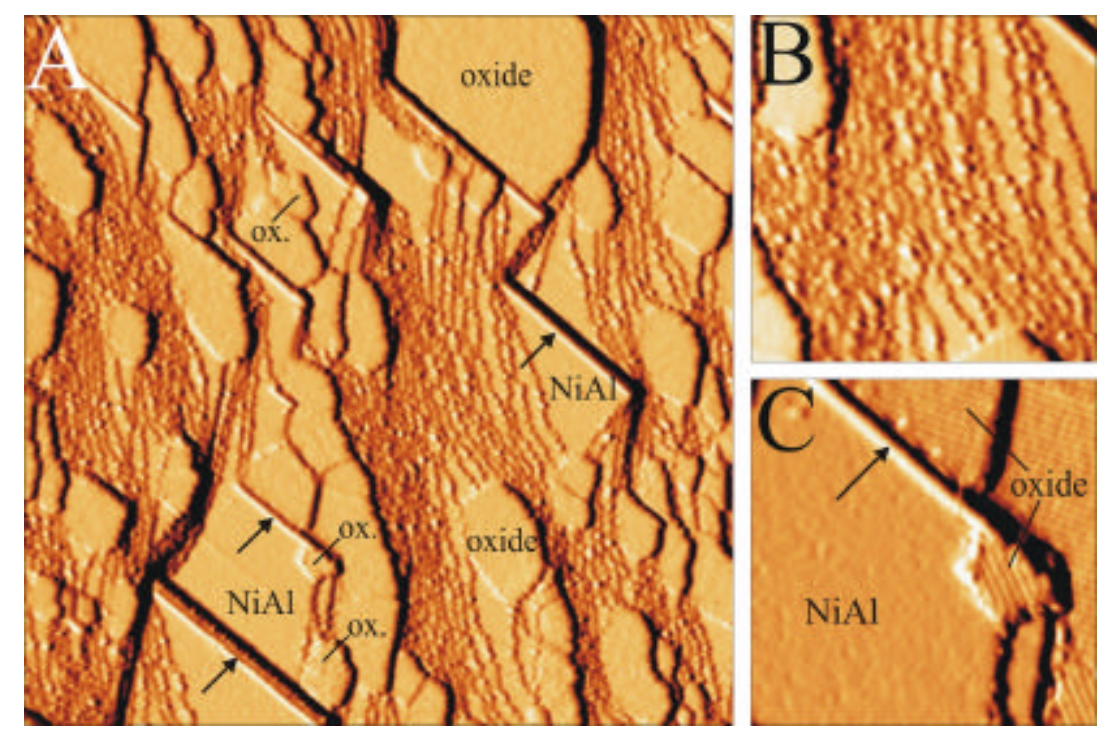

Fig.3

(A) Overview $(200 \times 200$ $\left.\mathrm{nm}^{2}\right)$ and $(\mathbf{B}, \mathbf{C})$ close-up images $\left(45 \times 45 \mathrm{~nm}^{2}\right)$ of $\mathrm{NiAl} \quad(16,14,1) \quad$ after exposure to $60 \mathrm{~L} \mathrm{O}_{2}\left(\mathrm{U}_{\mathrm{S}}=\right.$ 4.0 V, I $=0.1 \mathrm{nA})$. The images demonstrate the preferential formation of oxide nuclei (B) and small oxide patches (C) at the topside of step edges. Arrows mark protruding stripes with [001] orientation that often border large, triangular (110) planes.

The formation of an oxide nucleus at the topside of an edge leads to step pinning, because the oxide patch inhibits the participation of this region in the diffusive mass transport taking place at elevated temperatures. The global mass transport follows the miscut direction of the crystal, causing a backward motion of steps along the [111] direction and a decrease of the crystal tilt. As pinned steps are unable to take part in this movement, the adjacent terraces develop into large, triangular (110) planes, as observed on partly oxidized surfaces. In addition to these oxide nuclei, also the [001] oriented stripes (see arrows in Fig.3A) seem to be important for the development of triangular-shaped surface planes. The composition of theses one-dimensional features cannot be deduced from the STM data, however, their exclusive emergence after oxygen treatment suggests a distinct oxide phase that preferentially grows along the [001] direction. An oxide nature of these stripes would be compatible with their role as pinning lines for the triangular (110) planes. Oxide nano-rods of similar shape have previously been observed in LEEM studies performed during the initial oxidation of flat $\mathrm{NiAl}(110)$ surfaces [25]. Their one-dimensional growth has been traced back to an asymmetry in the lattice strain of this oxide phase $\left(\mathrm{NiAl}_{2} \mathrm{O}_{4}\right.$ as proposed by the authors), which favors elongation of the rod along the direction of smallest mismatch with the NiAl lattice, viz the [001] direction.

With increasing oxygen exposure, the number of oxide nuclei at the surface increases and pinning centers homogeneously arrange along the step edges (Fig.3B). The higher density 
of nucleation centers suppresses the development of extremely large (110) planes and leads to a smaller terrace-width distribution on the fully oxidized surface. The average terrace size has enlarged by a factor of 4-5 with respect to clean $\operatorname{NiAl}(16,14,1)$, which is accompanied with the agglomeration of monatomic steps into step bunches. Apparently, the expense to overcome step-step repulsion during bunching is compensated by the energy gain when growing large, step-free oxide patches. The mean terrace width of $8 \mathrm{~nm}$ on completely oxidized surfaces agrees surprisingly well with the typical distance between APDB boundaries on flat $\mathrm{NiAl}(110)$ [18]. As APDB are usually introduced into the oxide lattice to release the mismatch-induced strain, one might argue that this relaxation mechanism is replaced by the insertion of step edges on the vicinal surface. This mechanism would render the energy-expensive formation of domain boundaries unnecessary, thus explaining their absence in STM images taken for fully-oxidized $\operatorname{NiAl}(16,14,1)$. The mean terrace width on the vicinal surface now arises as the result of the energy balance between enlarging an oxide patch without step edge and stabilizing a single domain against the mismatch-induced lattice strain. From the fact that the observed terrace width nearly matches the APDB-distances on flat $\mathrm{NiAl}(110)$ follows that mainly stress and strain contribute to the final step arrangement and step-step repulsion plays only a minor role.
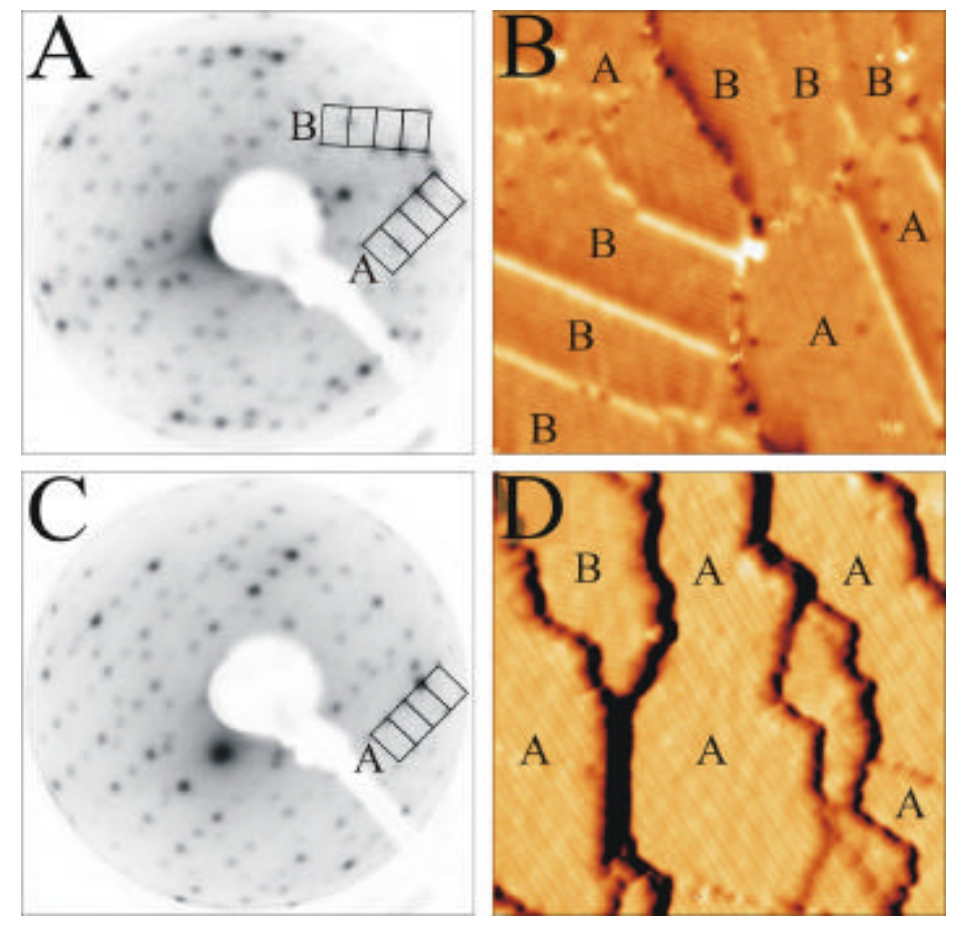

\section{Fig.4}

LEED and STM topographic images of $(\mathbf{A}, \mathbf{B}) \mathrm{NiAl}(110)\left(80 \times 80 \mathrm{~nm}^{2}\right)$ and $(\mathbf{C}, \mathbf{D}) \mathrm{NiAl}(16,14,1)(50 \times 50$ $\mathrm{nm}^{2}$ ) after oxidation with $1200 \mathrm{~L} \mathrm{O}_{2}$. Domains $\mathrm{A}$ and $\mathrm{B}$ are specified in the images. Both measurements conclusively show an equal domain distribution on $\mathrm{NiAl}(110)$, but a preferential formation of the A domain on the vicinal surface.

Finally, the influence of the regular step pattern on $\operatorname{NiAl}(16,14,1)$ on the domain distribution in the alumina film shall be discussed. On $\mathrm{NiAl}(110)$, the two reflection domains $\mathrm{A}$ and $\mathrm{B}$ are present with equal probability. The introduction of a step array with $34^{\circ}$ inclination against the [001] direction now creates an asymmetry regarding the two 
domains on the vicinal surface: The step edges roughly align with the short unit cell vector of type A domains that also marks the direction of the corresponding APDB (Fig.1). For the $B$ domains on the other hand, short unit cell vector and APDB's are inclined by almost $80^{\circ}$ against the step orientation. If APDB boundaries are indeed replaced by step edges for strain release in stepped alumina films, this mechanism should be more efficient for the A than for the B domain.

Already the LEED pattern visualizes the pronounced difference in the oxide domain distribution on flat and vicinal NiAl surfaces. On $\mathrm{NiAl}(110)$, the LEED reflexes follow two sets of lines that enclose an angle of $48^{\circ}$ and reflect the two alumina domains. Similar spot intensities in both sets indicate an equal occurrence of the $\mathrm{A}$ and $\mathrm{B}$ domain on the surface (Fig.4A) [26]. On $\mathrm{NiAl}(16,14,1)$, the LEED pattern is dominated by the rectangular symmetry of a single domain (Fig.4C). Reflexes that belong to its counterpart are strongly suppressed, suggesting the preferential growth of one domain type on the vicinal surface. STM measurements of fully oxidized $\operatorname{NiAl}(16,14,1)$ corroborate the spatially-averaged results of the LEED study. The two oxide domains are identified via their characteristic line pattern that follows the direction of the short unit-cell vector of the respective domain. The A domain, which is the one where APDB's align with the step orientation, is preferentially observed on the surface (Fig.4D). Statistical evaluation of STM images taken for different surface areas and preparation cycles yields a surface fraction of approximately 70-80\% covered with domain A, whereas type B domains are only present with $20 \%$.

This imbalance in the domain distribution supports our considerations on the influence of the step pattern on the probability to form either domain A or B. The lattice mismatch with the NiAl along [110] allows only 5-6 oxide unit cells (corresponding to 8-10 nm) to be grown without misfit dislocation to release the accumulated strain. For the A domain on $\mathrm{NiAl}(16,14,1)$, the dislocation line can be substituted by a step edge of similar orientation. Such relaxation mechanism is, however, ineffective for domain B, because (i) more than 56 unit cells are necessary to bridge a typical terrace, and (ii) the mean step direction on the $(16,14,1)$ surface is not compatible with the orientation of a type-B APDB. Consequently, B domains accumulate more stress when overgrowing the (110) planes of the vicinal NiAl surface, rendering their development thermodynamically less favourable [27]. Also from a kinetic point of view, A domains might be preferred. As one of the main crystallographic directions of A domains follows the initial step orientation on $\operatorname{NiAl}(16,14,1)$, the nucleation of this type might be facilitated in the early growth stage of the film. However, the observed domain distribution is more likely the result of thermodynamic and not kinetic growth conditions, as samples are thoroughly annealed after oxidation.

In conclusion, the preparation of thin alumina films on vicinal $\operatorname{NiAl}(16,14,1)$ is accompanied by strong modifications of the initial surface morphology. The sequence of regular (110) terraces transforms into large, triangular planes separated by step bunches. The new terrace width hereby corresponds to the characteristic domain size of the alumina 
film on flat $\mathrm{NiAl}(110)$, which identifies mismatch-induced lattice strain in the oxide layer as driving force for the structural modifications. The periodic step pattern on the $(16,14,1)$ surface favors the development of one reflection domain, for which a stress release via step edges is particularly efficient. The step edges on the vicinal alumina surface have a rather jagged and uneven character, which limits their utilization as template for the fabrication of ordered particle or nano-wire arrays. Straight oxide steps with a constant separation might, however, form on vicinal $\mathrm{NiAl}$ surfaces, where the miscut direction follows one of the $\mathrm{NiAl}$ lattice vectors.

[1] H. Brune, M. Giovannini, K. Bromann, K. Kern, Nature 394 (1998) 451.

[2] P.W. Murray, I. Brookes, S.A. Haycock, G. Thornton, Phys. Rev. Lett. 80 (1998) 988.

[3] N. Oncel, A. van Houselt, J. Huijben, A.S. Hallbäck, O. Gurlu, H. Zandvliet, B. Poelsema, Phys. Rev. Lett. 95 (2005) 116801.

[4] N. Nilius, E. Rienks, H.-P. Rust, H.-J. Freund, Phys. Rev. Lett. 95 (2005) 066101.

[5] A. Dmitriev, H. Spillmann, N. Lin, J.V. Barth, K. Kern, Angew. Chem. Int. Ed. 42 (2003) 2670.

[6] M. P. Pileni, J. Phys. Chem. 105 (2001) 3358.

[7] P. Gambardella, A. Dallmeyer, K. Maiti, M. C. Malagoli, S. Rusponi, P. Ohresser, W. Eberhardt, C. Carbone, K. Kern, Phys. Rev. Lett. 93, 077203 (2004).

[8] N. Neel, J. Kröger, R. Berndt, Appl. Phys. Lett. 88 (2006) 163101.

[9] F. Silly, M. Pivetta, M. Ternes, F. Patthey, J. Pelz, W. D. Schneider, Phys. Rev. Lett. 92 (2004) 016101.

[10] C. Becker, A. Rosenhahn, A. Wiltner, K. Bergmann, J. Schneider, P. Pervan, M. Milun, M. Kralj, K. Wandelt, New J. Phys. 4 (2002) 75.1.

[11] J. Kramer, C. Tegenkamp, H. Pfnür, Phys. Rev. B 67 (2003) 235401.

[12] S. Benedetti, P. Luches, S. Valeri, Surf. Sci. (2007) in press.

[13] R.M. Jaeger, H. Kuhlenbeck, H.-J. Freund, M. Wuttig, W. Hoffmann, R. Franchy, H. Ibach, Surf. Sci. 259 (1991) 235.

[14] M. Bäumer and H.-J. Freund, Prog. Surf. Sci. 61, 127 (1999).

[15] G. Kresse, M. Schmid, E. Napetschnig, M. Shishkin, L. Kohler, P. Varga, Science 308 (2005) 1440.

[16] S. Schauermann, V. Johánek, M. Laurin, J. Libuda, H.-J. Freund, Phys. Chem. Chem. Phys. 5 (2003) 5139.

[17] M. Sterrer, E. Fischbach, T. Risse, H.-J. Freund, Phys. Rev. Lett. 94 (2005) 186101.

[18] M. Kulawik, N. Nilius, H.-P. Rust, H.-J. Freund, Phys. Rev. Lett. 91, (2003) 256101.

[19] N. Nilius, M. Kulawik, H.-P. Rust, and H.-J. Freund, Phys. Rev. B 69 (2004) 121401.

[20] M. Schmid, M. Shishkin, G. Kresse, E. Napetschnig, P. Varga, M. Kulawik, N. Nilius, H.-P. Rust, H.-J. Freund, Phys. Rev. Lett. 97 (2006) 046101

[21] Another type of APDB runs along the diagonal of the unit cells of domain A and B, but occurs less frequent on the surface.

[21] L. Eierdal, F. Besenbacher, E. Lzegsgaard, I. Stensgaard, Surf. Sci. 312 (1994) 31.

[23] S. Hildebrandt, Ch. Hagendorf, T. Doege, Ch. Jeckstiess, R. Kulla, H. Neddermeyer, Th. Uttich, J. Vac. Sci. \& Technol.A 18 (2000) 1010.

[24] E. C. H. Sykes, P. Han, P. S. Weiss, J. Chem. Phys. 107 (2003) 5016.

[25] J. P. Pierce, K. F. McCarty, Phys. Rev. B 71, 125428 (2005).

[26] J. Libuda, M. Bäumer, H.-J. Freund, T. Bertrams,H. Neddermeyer, Surf. Sci. 318 (1994) 61.

[27] It should be emphasized that the conditions favoring the development of a single domain type apply only when $\operatorname{NiAl}(16,14,1)$ is completely oxidized. 